\title{
BIOENSAIOS COM OLIGOQUETAS EDÁFICOS PARA AVALIAÇÃO DA EFICÁCIA DO TRATAMENTO DE RESÍDUOS DE MINERAÇÃO DE CARVÃO EM CENÁRIO DE DISPOSIÇÃO TERRESTRE
}

\author{
BIOASSAYS WITH EARTHWORMS FOR EVALUATING THE EFFICIENCY ASSOCIATED WITH \\ THE TREATMENT OF COAL MINING RESIDUES DUE TO TERRESTRIAL DISPOSAL
}

\author{
Ricardo CESAR ${ }^{1}$, Bianca ROCHA ${ }^{2}$, Zuleica CASTILHOS ${ }^{2}$, Tácio de CAMPOS ${ }^{3}$, Claudio \\ SCHNEIDER ${ }^{2}$ \\ ${ }^{1}$ UFRJ, Departamento de Geografia. Email: ricardogc.geo@gmail.com \\ 22Centro de Tecnologia Mineral, CETEM. Emails: biancacrcr@gmail.com, zcastilhos@cetem.gov.br; claudio@cetem.gov.br \\ ${ }^{3}$ PUC-Rio, Depto de Engenharia Civil. Email tacio@puc-rio.br
}

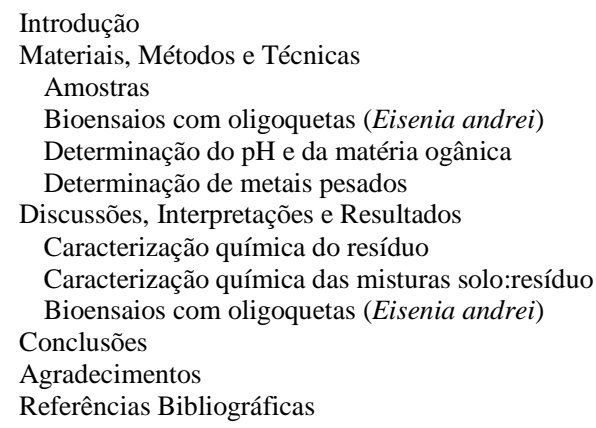

RESUMO - A oxidação da pirita, presente nos rejeitos e estéreis da mineração de carvão, pode desencadear uma drenagem ácida, induzindo uma redução drástica do $\mathrm{pH}$ dos solos e dos sistemas aquáticos vizinhos. O presente trabalho trata da avaliação da eficácia do processo de tratamento de um resíduo de mineração de carvão coletado na região carbonífera sul catarinense. O tratamento aplicado consistiu na separação das partículas conforme sua densidade, gerando três frações: pesada, mista e leve; com alto, intermediário e baixo teor de pirita, respectivamente. A eficácia do tratamento foi avaliada com base na quantificação de metais pesados e execução de bioensaios agudos e crônicos com oligoquetas edáficos (Eisenia andrei), conforme protocolos padrão (ISO). Os bioensaios foram somente aplicados à fração mista do resíduo, devido ao volume de material gerado com necessidade eventual de disposição terrestre. Misturas desta fração mista com um solo artificial foram preparadas nas seguintes proporções: 0, 6, 12, 24 e $50 \%$. Os resultados revelaram, de forma geral, uma redução das concentrações de metais pesados para a fração leve e atenuação do pH para as frações mista e leve, em comparação ao resíduo in natura. As concentrações de metais nas misturas de solo com a fração mista do resíduo estavam em concordância com os valores do CONAMA para qualidade de solos. Os bioensaios agudos com oligoquetas não resultaram em mortalidade nem em perda de biomassa dos animais, indicando baixa ecotoxicidade aguda. Contudo, os animais sobreviventes foram capazes de bioacumular elevadas quantidades de níquel na maior dose testada (50\%), um metal reconhecidamente tóxico aos oligoquetas. O bioensaio crônico indicou perda significativa de biomassa para a dose de $50 \%$, bem como redução significativa da reprodução para as doses de 24 e 50\%. Por fim, a fração mista do resíduo, de maior interesse do ponto de disposição terrestre, aparentemente não é tóxica aos oligoquetas edáficos em cenário de disposição em solo.

Palavras-chave: Oligoquetas, bioensaios, metais, toxicidade, mineração de carvão.

\begin{abstract}
The oxidation of pyrite in coal mining residues may cause acid drainage, degrading surrounding soils and aquatic ecosystems. This paper proposes the evaluation of a process for treating a coal mining residue in southern Santa Catarina State. Such treatment consisted of the separation of particles according to its density, thus generating three fractions: heavy, mixed and light ones, with high, intermediary and low content of pyrite, respectively. The efficiency of the process was evaluated through metal determination; and both acute and chronic bioassay with earthworms (Eisenia andrei), according to standard protocols. The bioassays were only applied to the mixed fraction, due to the large amount of material and eventual need for terrestrial disposal. Mixtures of the mixed fraction with an artificial soil were prepared for the following proportions: 0, 6, 12, 24 and 50\%. The results generally revealed the reduction of metal concentrations for the light fraction and attenuation of $\mathrm{pH}$ for the mixed and light fraction compared to the residue in natura. Metal concentrations in mixtures of mixed fraction and artificial soil were in agreement with the valued proposed by Brazilian law (CONAMA) for soil quality. The acute bioassays showed the absence of significant mortality and biomass loss, indicating low acute ecotoxicity. However, surviving animals bioaccumulated high amounts of nickel when exposed to the highest dosage of residue (50\%), a highly toxic metal for the earthworms. The chronic bioassay indicated significant biomass loss for the $50 \%$ dose, as well as significant reduction of the reproduction for the 24 and $50 \%$ doses. In conclusion, the mixed fraction of this residue is not apparently toxic to the earthworms in hypothetic scenarios of disposal in soil.
\end{abstract}

Keywords: Earthworms, bioassays, metals, toxicity, coal mining.

\section{INTRODUÇÃO}

A mineração de carvão, nos moldes até então executados no Brasil, tem trazido sérios impactos à saúde humana e ao ecossistema. A pirita, contida nos resíduos e estéreis do carvão, pode ser oxidada por exposição às águas pluviais e ao oxigênio, gerando o fenômeno da drenagem ácida de minas (DAM) (CETEM, 2000). 
A DAM se caracteriza por soluções contendo ácido sulfúrico capazes de decrescer substancialmente $\mathrm{o} \quad \mathrm{pH}$ dos solos e dos ecossistemas aquáticos vizinhos. A redução do $\mathrm{pH}$, por sua vez, pode induzir à ocorrência de mudanças na condutividade elétrica, acidez, oxigênio dissolvido, potencial redox e nas concentrações de ferro (Fe), alumínio (Al), manganês (Mn) e de metais pesados, sendo estes últimos lixiviados a partir dos resíduos e dos solos circunvizinhos à disposição dos resíduos (CETEM, 2000; Castilhos et al., 2010; Cesar et al., 2013).

A região carbonífera sul catarinense sofre há décadas com os impactos ambientais decorrentes da mineração de carvão (Castilhos et al., 2010, Cesar et al., 2013). A maior parte dos estudos geoambientais executados naquela região privilegiou a avaliação do comportamento hidrogeoquímico de contaminantes em bacias hidrográficas e águas subterrâneas, bem como a avaliação de risco ecológico associado aos organismos aquáticos (Cesar et al., 2013). Estudos focados nos impactos à saúde do ecossistema do solo ainda são escassos, sobretudo no que diz respeito à fauna edáfica. Neste sentido, a execução de bioensaios com organismos de solo é promissora, pois complementa os dados já existentes para organismos aquáticos e possibilita uma avaliação mais abrangente do risco ecológico associado à mineração (Cesar et al., 2013).

No caso dos solos, os oligoquetas edáficos (Eisenia andrei, Eisenia fetida) têm sido amplamente utilizados como organismos-teste na avaliação ecotoxicológica de solos contaminados, visto que possuem curto ciclo de vida, são simples de serem criados em laboratório e são de alta relevância ecológica servindo de alimento para diversas outras espécies terrestres (Neuhauser et al., 1985; Cesar et al., 2014). Além disso, a determinação dos teores de metais pesados nos tecidos dos oligoquetas sobreviventes dos ensaios possibilita a estimativa das frações potencialmente biodisponíveis daqueles elementos ao ecossistema do solo (Straalen et al., 2005).

O desenvolvimento de metodologias de remediação de resíduos de mineração de carvão é fundamental à minimização dos impactos negativos no sudeste de Santa Catarina. Neste sentido, o Centro de Tecnologia Mineral (CETEM-MCTI) tem contribuído para a geração deste tipo de tecnologia, com o apoio de seu campus avançado em Criciúma (SC). Contudo, a eficácia destas tecnologias deve ser monitorada, por exemplo, por meio de bioensaios utilizando organismos representativos do ecossistema, de modo a mensurar e prognosticar a redução efetiva de eventuais efeitos nocivos à saúde da biota terrestre.

Vale ressaltar que as análises químicas (totais ou parciais) em resíduos ou em solos impactados se referem somente à concentração de determinado agente tóxico no compartimento abiótico, o que não reflete obrigatoriamente a sua real toxicidade ao ecossistema. Assim, os bioensaios complementam as aná-lises químicas e oferecem suporte relevante à tomada de decisão em medidas de controle de poluição e de preservação da biodiversidade do solo.

O presente trabalho propõe a avaliação da eficácia de uma metodologia para recuperação de resíduos de mineração de carvão oriundos da região carbonífera sul catarinense, com base em bioensaios com oligoquetas (Eisenia andrei). As principais hipóteses de trabalho são: (i) o tratamento aplicado é eficiente no sentido de reduzir a geração de DAM e as concentrações de metais pesados; e (ii) a toxicidade aguda e crônica da fração mista do resíduo em misturas com solo artificial é baixa para os oligoquetas edáficos, em cenários de disposição terrestre.

\section{MATERIAIS, MÉTODOS E TÉCNICAS}

\section{Amostras}

O resíduo de mineração de carvão foi obtido na bacia carbonífera sul catarinense e foi submetido a um processo de tratamento realizado pela equipe do CETEM, que consistiu, basicamente, na separação das partículas conforme sua densidade. Para detalhes do tratamento, consulte Amaral-Filho et al. (2009).
Dessa forma, foram geradas três (3) frações: (i) leve (com baixo teor de pirita), (ii) mista (com teor intermediário de pirita) e (iii) pesada (com alto teor de pirita). A fração pesada não foi estudada do ponto de vista ecotoxicológico, visto que o objetivo é aproveitá-la na fabricação industrial de ácido sulfúrico. A fração leve corresponde, basicamente, a carvão e apresenta, 
reconhecidamente, menor toxicidade.

Portanto, no âmbito da avaliação ecotoxicológica, neste trabalho, são apresentados somente os resultados de toxicidade referentes à fração mista, de maior interesse ambiental devido não só ao grande volume de material gerado como também a necessidade frequente de disposição terrestre (ou seja, sobre o solo). Segundo Amaral-Filho et al. (2009), a fração mista deste resíduo tem textura predominantemente arenosa, apresenta teor de pirita em torno de $10 \%$ e, portanto, tem potencial para geração de DAM.

\section{Bioensaios com oligoquetas (Eisenia andrei)}

Para a realização dos bioensaios a fração mista do resíduo foi misturada com um solo artificial nas seguintes proporções: $0,6,12$, 24 e 50\%, sendo $0 \%$ composto apenas de solo puro. A composição do solo artificial segue as recomendações de Garcia (2004) e é constituído de $70 \%$ de areia quartzosa, $20 \%$ de caulim e $10 \%$ de pó de fibra de casca de coco.

O bioensaio agudo com oligoquetas da espécie Eisenia andrei foi executado com base nas recomendações de ISO (1998). O ensaio consistiu na exposição, em ciclos de 16 horas de luz e 8 horas de escuridão, de 10 oligoquetas adultos de peso semelhante (biomassa variando entre 300 e $700 \mathrm{mg}$ ) por réplica (4). Antes de serem introduzidos no solo, os oligoquetas foram deixados por 24 horas sobre papel absorvente umedecido para o esvaziamento do conteúdo intestinal.

Após 14 dias de exposição, verificou-se o número de organismos mortos. Os organismos sobreviventes são novamente deixados para o purgamento do conteúdo intestinal por 24 horas. Após esta etapa, os mesmos foram pesados para verificar a perda de biomassa, avaliada com base no peso médio inicial e final dos animais, isto é, antes e depois da exposição.

Por fim, os organismos foram congelados e enviados para determinação da concentração de metais pesados em seus tecidos. A biodisponibilidade de metais para os oligoquetas foi avaliada com base no cálculo do fator de bioconcentração, uma razão entre o teor total do metal em tecido animal pela concentração no solo (Liu et al., 2005).

O bioensaio crônico de reprodução com minhocas consistiu na exposição de organismos adultos, por 28 dias, às misturas de solo: resíduo, sob as mesmas condições laboratoriais do teste agudo. Ao final desta primeira etapa, os organismos adultos foram manualmente coletados, contados e pesados (ISO, 1998). Ao longo destes 28 dias, esterco moído foi servido uma vez por semana como alimento aos animais. Após a retirada dos animais adultos, o solo foi devolvido ao recipiente-teste e, após mais 28 dias, verificou-se o número de organismos jovens por réplica.

A contagem destes organismos foi auxiliada por um banho-maria colocado na base do recipiente-teste. Os juvenis foram retirados com auxílio de uma pinça e posteriormente contados.

\section{Determinação do pH e da matéria orgânica}

A determinação do $\mathrm{pH}$ foi realizada por meio de eletrodo combinado imerso em uma suspensão solo: solução de $\mathrm{KCl}(1 \mathrm{~N})$ na proporção 1:2,5, conforme EMBRAPA (1997).

O teor de matéria orgânica foi determinado com base na pesagem de 0,25 g de amostra em barquinha de porcelana, seguido de lixiviação com $\mathrm{HCl}$ 50\% por 15 horas (visando a destruição do carbono não orgânico). Após a remoção da solução ácida, o resíduo obtido foi então lavado com água destilada e deixado sob banho de luz para secagem, por 15 horas. Por fim, o resíduo foi queimado em forno a $1500^{\circ} \mathrm{C}$, com o uso do equipamento Leco SNS-2000, para quantificação do teor de carbono orgânico total (COT).

\section{Determinação de metais pesados}

A determinação da concentração total de metais pesados ( $\mathrm{Zn}, \mathrm{Cu}, \mathrm{Pb}, \mathrm{Ni}$ e $\mathrm{Cd}$ ) em amostras bióticas e abióticas foi realizada com base na solubilização de $1 \mathrm{~g}$ de amostra em uma solução ácida composta de $2 \mathrm{HF}: 1 \mathrm{HClO}_{4}: 1 \mathrm{HNO}_{3}$, em bécher de platina.

Após a secagem em chapa de aquecimento, a solução é retomada com o emprego de ácido nítrico 5\% $\left(\mathrm{HNO}_{3}\right)$ para posterior análise em Espectrometria de Emissão com Plasma Indutivamente Acoplado (ICP-OES).

Antes de serem analisados, os oligoquetas sobreviventes dos testes foram previamente congelados, liofilizados, triturados e homogeneizados.

A quantificação dos metais pesados foi realizada nas frações leve e mista do resíduo, bem como no resíduo puro antes do tratamento (“fração alimentação”). 
A avaliação do incremento da concentração de metais com o aumento de dose de resíduo no solo foi avaliada com base no cálculo dos fatores de contaminação (FC), uma razão entre o teor do metal no solo puro (sem adição de resíduo) pela concentração no solo contaminado (misturado com resíduo). Valores maiores do que uma (1) unidade indicam incremento na concentração de metais.

A avaliação do grau de poluição de metais na fração mista do resíduo foi efetuada com base no cálculo do Índice de Geoacumulação (IGEO), descrito em Müller (1979), que consiste em uma escala logarítmica que compara a concentração de metal obtida no resíduo com o teor reportado para o folhelho médio (Turekian \& Wedepohl, 1961). O IGEO é agrupado em 7 classes (de 0 a 6), onde zero (0) corresponde a ausência de poluição e seis (6) representa o nível máximo de poluição.

\section{RESULTADOS E DISCUSSÃO}

\section{Caracterização química do resíduo}

A tabela 1 apresenta a determinação de parâmetros físico-químicos e os teores totais de metais obtidos em distintas frações do resíduo estudado. $\mathrm{O}$ resíduo in natura (fração alimentação) apresentou, como esperado, valor de $\mathrm{pH}$ mais baixo em comparação às frações mista e leve. Esta constatação indica que de fato o tratamento aplicado foi capaz de diminuir o teor de pirita para as frações mista e leve, resultando na geração de uma DAM menos intensa (ou seja, uma solução de $\mathrm{pH}$ menos ácido).

O teor de COT na fração leve foi elevado (37\%), valor este até quatro vezes maior do que àqueles encontrados na fração alimentação e mista. Vale ressalvar que a fração leve apresenta baixa densidade e é majoritariamente composta por carvão (ou seja, matéria orgânica), fato este que justifica o alto valor encontrado para o COT.

Os teores de $\mathrm{Fe}, \mathrm{Al}$ e $\mathrm{Mn}$ foram menores (até duas vezes menores para o $\mathrm{Fe}$ ) nas frações leve em comparação às frações mista e alimentação. Minerais de $\mathrm{Fe}$, $\mathrm{Al}$ e $\mathrm{Mn}$ são, em geral, de elevada densidade e, portanto, é válido, de fato, supor que a ocorrência dessas assembleias minerais na fração leve seja menor.

Além disso, o Fe associa-se à ocorrência de pirita, cuja concentração aparentemente também diminuiu na fração leve (conforme indicado pelo aumento do $\mathrm{pH}$ ). As concentrações de $\mathrm{Cd}$ ficaram abaixo do limite de detecção $(0,01 \mathrm{mg} / \mathrm{kg})$ nas frações alimentação, mista e leve e, por essa razão, não aparecem na tabela 1 .

Com exceção do $\mathrm{Cr}$, as concentrações de metais pesados na fração leve do resíduo foram menores se comparadas aos teores encontrados nas frações alimentação e mista, o que supostamente ratifica a eficiência química do processo de remediação aplicado.

Vale ressaltar que os metais pesados apresentam elevada densidade e possuem forte afinidade com os sulfetos e, portanto, é de se esperar que suas concentrações na fração leve também sejam mais baixas.

Por outro lado, é importante ressaltar que o Zn apresentou sua mais elevada concentração na fração mista (valor até duas vezes maior do que aquele determinado na alimentação e na fração leve).

O cálculo dos IGEOs para a fração mista do resíduo indicou que todos os metais se encontram na Classe 0 ("praticamente nãopoluído”), a exceção do $\mathrm{Pb}$ que ficou na Classe 1 ("pouco a moderadamente poluído").

Do ponto de vista toxicológico, o referido valor de IGEO para o $\mathrm{Pb}$ e a elevada concentração de Zn obtida na fração mista merecem posição de maior atenção, devido à reconhecida toxicidade destes metais (Lukkari et al., 2005; Savard et al., 2007).

Contudo, de forma geral, as concentrações de metais diminuíram apenas para a fração leve (exceto $\mathrm{Cr}$ ), ratificando parcialmente a primeira hipótese de trabalho, tendo em vista que o decréscimo das concentrações de metais da fração alimentação para a mista não foi importante.

Outra constatação importante a ser ressaltada é que as concentrações de metais nas distintas frações se encontram muito acima dos valores encontrados por Cesar et al. (2013), que estudaram uma amostra de resíduo coletado em pilha ao céu aberto na bacia do Rio Urussanga (sul de Santa Catarina).

Entretanto, apesar das concentrações de metais terem sido mais baixas, Cesar et al. (2013) relataram a ocorrência de toxicidade do resíduo para Lactuca sativa (alface) e Daphnia similis (cladócero de água doce). 
Tabela 1 - Metais pesados e parâmetros físico-químicos das frações alimentação, mista e leve do resíduo de mineração de carvão e valores obtidos em resíduo estudado por Cesar et al. (2013). COT = carbono orgânico total.

\begin{tabular}{c|c|c|c|c}
\hline Parâmetros & Alimentação & Fração Mista & Fração Leve & Cesar et al., (2013) \\
\hline $\mathrm{pH}\left(\mathrm{H}_{2} \mathrm{O}\right)$ & 3,80 & 4,60 & 4,71 & 3,40 \\
\hline $\mathrm{COT}(\%)$ & 8,1 & 6,6 & 37,2 & - \\
\hline $\mathrm{Al}(\%)$ & 11,1 & 11,0 & 9,5 & - \\
\hline $\mathrm{Fe}(\%)$ & 4,4 & 4,1 & 2,2 & - \\
\hline $\mathrm{Mn}(\mathrm{mg} / \mathrm{kg})$ & 72,0 & 73,1 & 53,5 & 0,06 \\
\hline $\mathrm{Zn}(\mathrm{mg} / \mathrm{kg})$ & 62,4 & 129,0 & 58,7 & 2,70 \\
\hline $\mathrm{Cu}(\mathrm{mg} / \mathrm{kg})$ & 28,5 & 27,0 & 21,6 & 36,40 \\
\hline $\mathrm{Pb}(\mathrm{mg} / \mathrm{kg})$ & 61,4 & 57,1 & 48,5 & - \\
\hline $\mathrm{Ni}(\mathrm{mg} / \mathrm{kg})$ & 20,5 & 21,2 & 18,4 & 30,80 \\
\hline $\mathrm{Cr}(\mathrm{mg} / \mathrm{kg})$ & 50,9 & 50,8 & 58,4 & \\
\hline
\end{tabular}

Caracterização química das misturas solo: resíduo

A tabela 2 apresenta a determinação do $\mathrm{pH}$, da capacidade de retenção de água (CRA) e as concentrações totais de metais obtidas nas misturas de diferentes proporções de solo artificial com a fração mista do resíduo. Os dados sugerem a redução do $\mathrm{pH}$ das misturas com o incremento de dose, sugerindo que adição do resíduo ao solo foi capaz de acidificá-lo, o que indicaria a ocorrência de DAM. A CRA, por outro lado, aumenta com o incremento de dose, uma provável consequência da textura arenosa do resíduo.

As concentrações de metais nas misturas solo: resíduo estão em concordância com valores propostos pela Resolução 420 do CONAMA (2009), que estabelece as dire- trizes para a qualidade de solos no Brasil. Por outro lado, as concentrações de Zn (nas doses de 24 e 50\%) e de $\mathrm{Pb}$ (nas desses de 12, 24 e $50 \%$ ) excederam o valor referência proposto por CETESB (2014), que diz respeito ao background de geoquímica de solos do Estado de São Paulo.

Os FCs indicam que houve incremento da concentração de metais com o aumento de dose de resíduo no solo. Contudo, tais incrementos foram aparentemente maiores para o $\mathrm{Ni}$ e o $\mathrm{Cr}$. No caso destes metais, tais valores chegam a indicar um incremento de até 4 vezes da concentração do metal. Estes resultados merecem posição de maior atenção, devido à toxicidade dos elementos supracitados e do elevado incremento relativo com o aumento de dose de resíduo no solo.

Tabela 2 - Metais pesados e parâmetros físico-químicos nas misturas de solo artificial com a fração mista do resíduo de mineração de carvão. $\mathrm{CRA}=$ capacidade de retenção de água. $\mathrm{SL}=$ concentração no solo em mg $/ \mathrm{kg}$. FC = fator de contaminação.

\begin{tabular}{|c|c|c|c|c|c|c|c|c|c|c|c|c|}
\hline \multirow{2}{*}{ Doses (\%) } & \multirow{2}{*}{$\mathbf{p H}$} & \multirow{2}{*}{$\begin{array}{l}\text { CRA } \\
(\%)\end{array}$} & \multicolumn{2}{|c|}{$\mathbf{Z n}$} & \multicolumn{2}{|c|}{$\mathbf{C u}$} & \multicolumn{2}{|c|}{$\mathbf{P b}$} & \multicolumn{2}{|c|}{$\mathbf{N i}$} & \multicolumn{2}{|c|}{$\mathrm{Cr}$} \\
\hline & & & \begin{tabular}{l|l|} 
SL &
\end{tabular} & FC & SL & FC & SL & FC & SL & FC & SL & FC \\
\hline 0 & 5,61 & 68,57 & 48,60 & - & 6,80 & - & 13,00 & - & 2,90 & - & 7,20 & - \\
\hline 6 & 5,36 & 69,91 & 53,42 & 1,10 & 8,01 & 1,18 & 15,65 & 1,20 & 4,00 & 1,38 & 9,82 & 1,36 \\
\hline 12 & 4,93 & 61,70 & 58,25 & 1,20 & 9,22 & 1,36 & $18,29 *$ & 1,41 & 5,10 & 1,76 & 12,43 & 1,73 \\
\hline 24 & 4,77 & 54,59 & $67,90 *$ & 1,40 & 11,65 & 1,71 & $23,58 *$ & 1,81 & 7,29 & 2,51 & 17,66 & 2,45 \\
\hline 50 & 4,60 & 51,81 & $88,80^{*}$ & 1,83 & 16,90 & 2,49 & $35,05^{*}$ & 2,70 & 12,05 & 4,16 & 29,00 & 4,03 \\
\hline \multicolumn{3}{|c|}{ Referência (CETESB, 2014) (mg/kg) } & \multicolumn{2}{|c|}{60} & \multicolumn{2}{|c|}{35} & \multicolumn{2}{|c|}{17} & \multicolumn{2}{|c|}{13} & \multicolumn{2}{|c|}{40} \\
\hline \multicolumn{3}{|c|}{$\begin{array}{c}\text { Prevenção (CONAMA, 2009) } \\
\text { (mg/kg) }\end{array}$} & \multicolumn{2}{|c|}{300} & \multicolumn{2}{|c|}{60} & \multicolumn{2}{|c|}{72} & \multicolumn{2}{|c|}{30} & \multicolumn{2}{|c|}{75} \\
\hline \multicolumn{3}{|c|}{$\begin{array}{c}\text { Intervenção (CONAMA, 2009) } \\
\text { (mg/kg) }\end{array}$} & \multicolumn{2}{|c|}{450} & \multicolumn{2}{|c|}{200} & \multicolumn{2}{|c|}{180} & \multicolumn{2}{|c|}{70} & \multicolumn{2}{|c|}{150} \\
\hline
\end{tabular}

\section{Bioensaios com oligoquetas (Eisenia andrei)}

A tabela 3 apresenta as variações de biomassa de E. andrei e os valores de FBC obtidos para as misturas de solo artificial e fração mista do resíduo, após 14 dias de exposição. Os resultados desses bioensaios agudos revelaram a ausência significativa de mortalidade e de perda de biomassa dos 
organismos testados, indicando baixa ecotoxicidade aguda para os oligoquetas e corroborando a segunda hipótese de trabalho.

A determinação dos teores de metais nos tecidos dos animais e seus respectivos fatores de bioconcentração (FBC) indicaram baixa captação biológica de metais altamente tóxicos (FBC < 0,5), como por exemplo, no caso de $\mathrm{Pb}$ e $\mathrm{Cr}$, em concordância com a baixa toxicidade observada.

$\mathrm{O}$ valor de $\mathrm{FBC}$ obtido para o $\mathrm{Ni}$ na maior dose testada (50\%) foi maior do que uma (1) unidade, indicando que houve bioacumulação e que, portanto, deve ser melhor investigada. Os FBCs obtidos para o Zn ficaram acima de uma (1) unidade para algumas doses, inclusive para o solo artificial puro $(0 \%)$. Porém, observa-se o decréscimo dos FBCs de Zn e do $\mathrm{Cr}$ com o incremento.

No caso do Zn, vale salientar que este é um metal essencial ao metabolismo dos oligoquetas e desempenha papel importante no crescimento e regeneração dos tecidos desses invertebrados (Lukkari et al., 2005).

Uma vez que o Zn é um metal essencial, é válido supor que o mesmo possua rotas de absorção facilitadas. O Cu, apesar de também ser um metal essencial, apresentou valores muito baixos de FBC, indicando baixa biodisponibilidade sob as condições testadas.

Prováveis hipóteses para a baixa biodisponibilidade de $\mathrm{Cu}$ e $\mathrm{Cr}$, e para a redução dos FBCs de Zn e Cr com o incremento de dose de resíduo no solo podem estar associadas a: (i) mecanismos de saturação pelos organismos, isto é, o organismo já acumulou o máximo tolerável pelo metabolismo e, por esta razão, passa a depurar o metal.

Esta hipótese justifica, em parte, a obtenção de um FBC acima de uma unidade no solo artificial puro (0\%) para o $\mathrm{Zn}$, tendo em vista que os oligoquetas já contam naturalmente com altas reservas metabólicas de $\mathrm{Zn}$ para futuras demandas fisiológicas (Lukkari et al., 2005); e (ii) boa parte dos metais podem estar retidos na estrutura cristalina dos grãos do resíduo e, portanto, pouco disponíveis para captação biológica.

Cesar et al. (2013), ao estudarem a toxicidade aguda de um resíduo de mineração de carvão (oriundo da região carbonífera sul catarinense) em misturas com solos artificiais, também encontraram níveis baixos de mortalidade e de perda de biomassa de oligoquetas, bem como baixa biodisponibilidade de metais.

Naquela ocasião, os autores atribuíram à baixa toxicidade ao fato de que os resíduos se encontravam ao céu aberto, e boa parte dos metais já havia sido lixiviada. Ainda, os referidos autores também reportaram altos valores de $\mathrm{FBC}$ para o $\mathrm{Zn}$ e o $\mathrm{Cu}$, e os justificaram com base na essencialidade desses metais à fisiologia dos oligoquetas.

No caso do presente estudo, em contradição a Cesar et al. (2013), os FBCs para $\mathrm{Cu}$ foram baixos e não indicaram bioacumulação do metal.

Esta baixa biodisponibilidade de $\mathrm{Cu}$ em comparação ao resíduo estudado por Cesar et. al (2013) pode, novamente, estar associada deste metal estar potencialmente presente na forma de assembleias minerais e, portanto, geoquimicamente pouco disponíveis.

Castilhos et al. (2010) determinaram o pH e as concentrações de metais em solos aluviais impactados pela atividade da mineração na região carbonífera sul catarinense. Tais valores foram semelhantes àqueles encontrados nas misturas solo: resíduo deste presente estudo.

Por outro lado, Castilhos et al. (2010) indicaram a ocorrência de toxicidade aguda (morte e perda de biomassa) para Eisenia andrei em algumas amostras, em correlação positiva com o decréscimo do $\mathrm{pH}$.

Esta constatação realça a importância do uso de solos locais na avaliação da ecotoxicidade, tendo em vista que parâmetros como $\mathrm{pH}$, Eh, condutividade elétrica, mineralogia das argilas, oxi-hidróxidos de ferro e alumínio, matéria orgânica, dentre outros, desempenham papel de suma importância na toxicidade e na mobilidade geoquímica da contaminação (Rodrigues-Filho \& Maddock, 1997; Matzke et al., 2008; Cesar et al., 2014; Cesar et al., 2015).

Esta avaliação preliminar da toxicidade do resíduo, em solos artificiais, faz-se importante para comparação e padronização de resultados servindo como screening e apoio ao estabelecimento de estratégias analíticas a serem adotadas em ensaios com solos reais.

A figura 1 apresenta os resultados dos bioensaios crônicos com E. andrei nas misturas entre o solo e a fração mista do resíduo. Os resultados indicam redução significativa da biomassa dos animais adultos para dosagem de $50 \%$ após 28 dias de 
exposição, sugerindo que os animais estavam em condições de estresse. Os dados ainda indicam redução significativa da reprodução para as duas maiores dosagens testadas $(24 \mathrm{e}$ $50 \%$ ), apontando um eventual comprometimento da perpetuação da espécie.

Tabela 3 - Variações da biomassa de Eisenia andrei e fatores de bioconcentração (FBC) de metais obtidos em bioensaios agudos utilizando solos artificiais tratados com diferentes doses da fração mista do resíduo de mineração de carvão.

\begin{tabular}{c|c|c|c|c|c|c}
\hline Doses (\%) & Biomassa (\%) & $\mathbf{Z n}$ & $\mathbf{C u}$ & $\mathbf{P b}$ & $\mathbf{N i}$ & $\mathbf{C r}$ \\
\hline 0 & $91,00 \pm 1,07$ & 1,58 & 0,22 & 0,15 & 0,10 & 0,49 \\
\hline 6 & $93,03 \pm 2,20$ & 1,14 & 0,35 & 0,13 & 0,08 & 0,29 \\
\hline 12 & $89,23 \pm 2,54$ & 0,72 & 0,20 & 0,11 & 0,06 & 0,18 \\
\hline 24 & $88,60 \pm 3,63$ & 1,26 & 0,10 & 0,08 & 0,04 & 0,12 \\
\hline 50 & $91,99 \pm 4,05$ & 0,83 & 0,30 & 0,06 & 1,12 & 0,06 \\
\hline
\end{tabular}

Contudo, vale ressaltar que a ocorrência significativa de toxicidade crônica foi detectada somente para as misturas com dosagens mais elevadas de resíduo. Porém, esta toxicidade pode ter sido induzida pela escassez de alimento (mesmo considerando o esterco servido durante o teste, já que este foi igualmente adicionado a todos os tratamentos) ou por mudanças na estrutura física do solo, e não necessariamente pela presença de metais ou pela geração de DAM. Estes resultados devem ser melhor investigados com base em testes de comportamento de fuga com oligoquetas e, sobretudo, com testes crônicos utilizando outros invertebrados de solo (por exemplo, colêmbolos) de forma a gerar um prognóstico mais amplo e consistente acerca do risco ecotoxicológico.

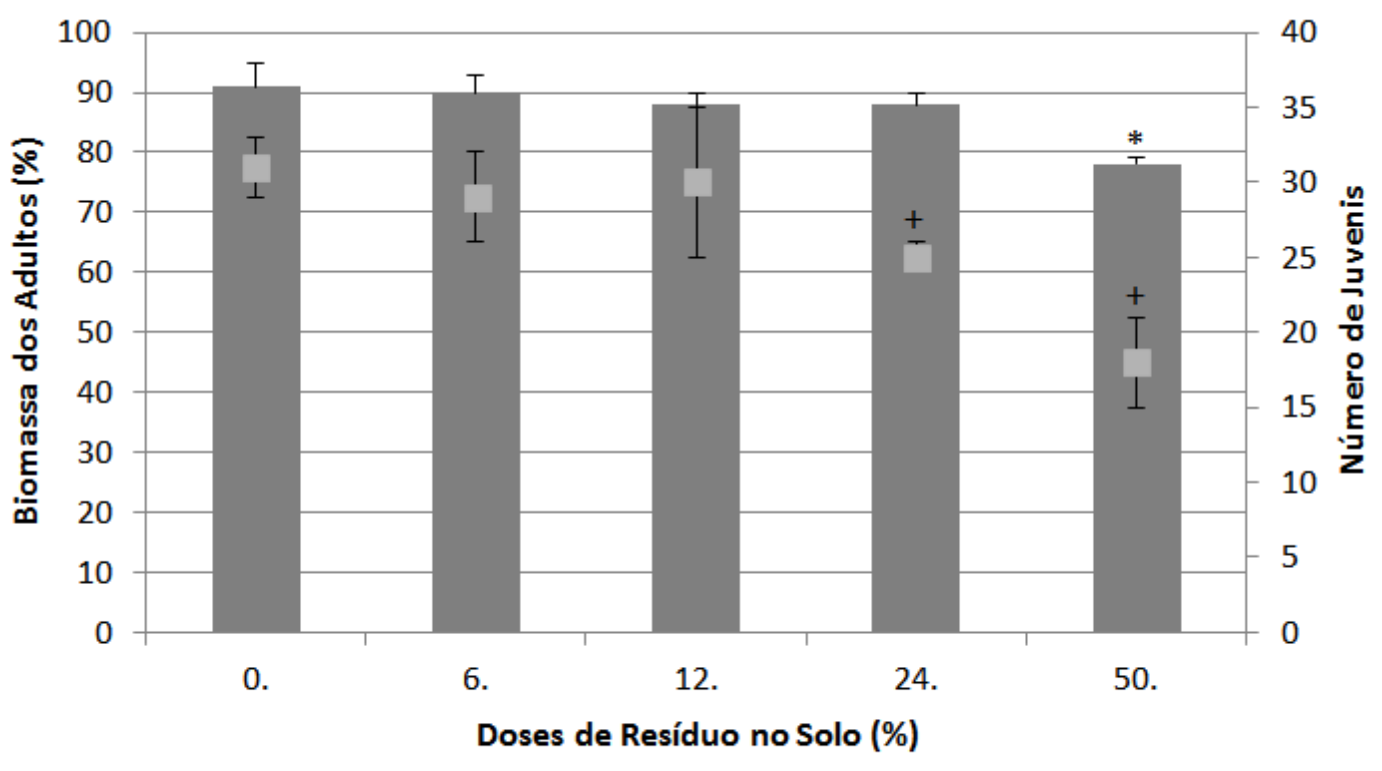

- Biomassa Reprodução

Figura 1- Variações da biomassa dos organismos adultos (após 28 dias de exposição) e número de juvenis obtidos em bioensaio crônico de reprodução com Eisenia andrei utilizando misturas de diferentes proporções de solo artificial e a fração mista do resíduo de mineração de carvão. * e + = biomassa e reprodução, respectivamente, diferentes do controle ( $<$ < 0,05; Kruskall-Wallis).

\section{CONCLUSÕES}

Os teores totais de metais determinados nas misturas de solo artificial com a fração mista do resíduo foram relativamente baixos e estão em conformidade com os padrões de qualidade de solos preconizados pelo CONAMA. A toxicidade aguda desta fração mista em misturas com solo artificial foi baixa, mesmo para dosagens elevadas (50\%).

Contudo, vale ressaltar que os organismos foram capazes de bioacumular $\mathrm{Ni}$ na maior 
dose testada, fato este que deve ser melhor estudado. Do ponto de vista crônico, a ocorrência de toxicidade somente foi detectada para as maiores doses testadas.

Embora, a princípio, a fração mista não tenha sido significativamente tóxica aos oligoquetas terrestres, tais resultados devem ser confirmados com ensaios utilizando outros invertebrados de solos e organismos aquáticos. Além disso, é recomenda-se a execução de ensaios com o emprego de solos naturais representativos da pedologia regional do sul de SC, de forma a estudar a influência potencial das propriedades do solo sobre a ecotoxicidade e a biodisponibilidade de metais para a biota edáfica.

\section{AGRADECIMENTOS}

Os autores gostariam de agradecer à Coordenação de Análises Minerais (COAM) do Centro de Tecnologia Mineral (CETEM/MCTI) pelo suporte na determinação de metais pesados. $\mathrm{O}$ apoio financeiro do CNPq e CAPES, através de concessão de bolsa a Ricardo Cesar (Pósdoutorado/PNPD - CAPES) e a Bianca Rocha (IC - CNPq) foi de fundamental importância à realização deste trabalho.

\section{REFERÊNCIAS}

AMARAL-FILHO, J.; SCHNEIDER, I.V.H.; BRUM, I.A.S; MILTZAREK, G.; SAMPAIO, C.H.; SCHNEIDER, C.H. Caracterização dos rejeitos de carvão do módulo B da carbonífera Criciúma S.A. In: ENCONTRO NACIONAL DE TRATAMENTO DE MINÉRIOS E METALURGIA EXTRATIVA, XXIII, 2009. Gramado Anais ...Gramado: CD-ROOM.

CASTILHOS, Z.C.; BIDONE, E.D.; CESAR, R.G.; EGLER, S.G., BIANCHINNI, M., ALEXANDRE, N.Z.; NASCIMENTO, T. Metodologia de monitoramento da qualidade das águas da Bacia Carbonífera Sul Catarinense: ferramenta para gestão em poluição ambiental. Série Gestão e Planejamento Ambiental. Rio de Janeiro: CETEM, v.1, p. 03-105, 2010.

CESAR, R.G.; COELHO, M.B.; ALVARO, T.T.; COLONESE, J.P.; CASTILHOS, Z.C.; EGLER, S.G.; BIDONE, E.D.; POLIVANOV, H.; ALEXANDRE, N.Z. Disposição continental de resíduos de mineração de carvão: drenagem ácida, ecotoxicidade aguda e biodisponibilidade de metais. Ecotoxicology and Environmental Contamination, v. 8, p. 17-22, 2013

CESAR, R. G.; NATAL-DA-LUZ, T; BIDONE E. D.; CASTILHOS, Z.; POLIVANOV, H.; SOUSA, J. P. Disposal of dredged sediments in tropical soils: ecotoxicological evaluation based on bioassays with springtails and enchytraeids. Environmental Science and Pollution Research, v. 22, p. 2916-2924, 2015.

CESAR, R. G.; NATAL-DA-LUZ, T.; SOUSA, J. P. F.; COLONESE, J.; BIDONE, E. D.; CASTILHOS, Z. C.; EGLER, S .G.; POLIVANOV, H. Disposal of dredged sediments in tropical soils: ecotoxicological effects on earthworms. Environmental Monitoring and Assessment, v. 186, p. $1487-1497,2014$

CETEM/CANMET.2000. Projeto Conceitual Preliminar para Recuperação Ambiental da Bacia Carbonífera Sul Catarinense. Relatório Técnico elaborado para o SIECESC (RT33/2000), CETEM/MCTI.

CETESB (Companhia de Tecnologia de Saneamento Ambiental). 2014. In: Valores Orientadores para Solos e Águas Subterrâneas no Estado de São Paulo. Disponível em: < http://cetesb.sp.gov.br/solo/wp-content/uploads/sites/34/2013 /12/VO-2014.pdf> Accesso em 02 Mar. 2016

CONAMA. 2009. Resolução 420. Disp. em: $<$ http://www.mma.gov.br/port/conama/legiabre.cfm?codlegi= 506>. Acesso em 19 Out. 2010

EMBRAPA (EMPRESA BRASILEIRA DE PESQUISA AGROPECUÁRIA). 1997, Centro Nacional de Pesquisa de
Solos. In: Manual de Métodos de Análises de Solo. Rio de Janeiro, RJ, pp. 212.

GARCIA, M. Effects of pesticides on soil fauna: Development of ecotoxicology test methods for tropical regions. In: VLEK, P.L.G. (Ed.). Ecology and Development Series, v. 19, Cuvillier Verlag Gottingen, 282p., 2004.

ISO (International Organization for Standardization) 1998. Soil quality - Effects of pollutants on earthworms (Eisenia fetida) - Part 2: Determination of effects on reproduction. ISO 11268-2. Geneve, Switzerland.

LIU, X., CHENGXIAO, H., ZHANG, S. Effects on earthworm activity on fertility and heavy metals bioavailability in sewage sludge. Environment International, v.31, p.874-879, 2005.

LUKKARI, T.; ASTSINKI, M.; VÄISÄNEN, A.; HAIMI, J. Toxicity of copper and zinc assessed with three different earthworms tests. Applied Soil Ecology, v.30, p.133-146, 2005. MATZKE, M.; STOLTE, S.; ARNING, J.; UEBERS, U.; FILSER, J. Imidazolium based ionic liquids in soils: effects of the side chain length on wheat (Triticum aestivum) and cress (Lepidium sativum) as affected by different clays and organic matter. Green Chemistry, v.10, p.584-591, 2008.

MÜLLER, G. Schwermetalle in den Sedimenten des Rheins Veränderungenseit. Umschau, v.79, p.778-783, 1979.

NEUHAUSER, E. F.; LOEHR, R. C.; MILLIGAN, D. L.; MALECKI, M. R. Toxicity of metals to the earthworm Eisenia fetida. Biology and Fertility of Soils, v.1, p.149-152, 1985.

RODRIGUES-FILHO, S.; MADDOCK, J.E.L. Mercury pollution in two gold mining areas of the Brazilian Amazon. Journal of Geochemical Exploration, v. 58, p. 231-240, 1997.

SAVARD, K.; BERTHELOT, Y.; AUROY, A.; SPEAR, P.A.; TROTTIER, B.; ROBIDOUX, P.Y. Effects of HMX-Lead Mixtures on Reproduction of the Earthworm Eisenia andrei. Archives of Environmental Contamination and Toxicology, v. 53, p. 351-358, 2007.

STRAALEN, N. M.; DONKER M. H.; VIJVER, M. G.; GESTEL, C. A. M. Bioavailability of contaminants estimated from uptake rates into soil invertebrates. Environmental Pollution, v.136, p. 409-417, 2005.

TUREKIAN, K. K. \& WEDEPOHL, K. H. Distribution of the elements in some major units of the Earth's crust. Geological Society American Bulletin, v. 72, p. 175-192, 1961.

Submetido em 15 de março de 2016 Aceito em 14 de março de 2017 